\title{
TUMOR PRIMARIO QUÍSTICO DE LA ÓRBITA: A PROPÓSITO DE UN CASO DE HIDATIDOSIS INFANTIL
}

\author{
Deivy Cruzado-Sánchez ${ }^{1, a}$, Sandra Salas-Diaz ${ }^{2, b}$, Walter A. Tellez ${ }^{2, b}$, Grisnery Maquera-Torres ${ }^{1, c}$, \\ Solon Serpa-Frias ${ }^{1, a}$
}

\begin{abstract}
RESUMEN
Los tumores quísticos primarios de la órbita, causados por infestaciones parasitarias como el Equinococcus granulosus son raros. Al ser el Perú zona endémica para este parásito, presentamos el caso de una niña de 10 años de edad, proveniente de una zona rural de Huancavelica, con proptosis axial irreductible del globo ocular derecho, de curso progresivo, con pérdida de la agudeza visual e imágenes tomográficas de la órbita en relación a masa tumoral de apariencia quística. Los exámenes serológicos fueron negativos, la paciente fue sometida a resección quirúrgica con resultado histopatológico de membranas anhistas correspondientes a quiste hidatídico. Al año de seguimiento no se evidencia recurrencia local ni sistémica. En conclusión, ante una tumoración orbitaria quística en pacientes pediátricos, se debe de considerar como diagnóstico diferencial la hidatidosis orbitaria, sobre todo si proceden de zonas endémicas.
\end{abstract}

Palabras clave: Órbita; Tumor; Neoplasias orbitárias; Equinococcus granulosus; Equinococosis; Zoonosis (fuente: DeCS BIREME).

\section{PRIMARY ORBITAL CYSTIC TUMOR: A CASE OF HYDATIDOSIS IN A CHILD}

\begin{abstract}
Primary orbital cystic tumors caused by Echinococcus granulosus are rare. As this parasite is endemic in Peru, we report the case of a 10-year-old girl from a rural area of Huancavelica, who presented with progressive and irreducible axial proptosis of the right eye and loss of vision. Orbital tomographic images demonstrated a tumor mass with a cystic appearance. Serologic tests were negative. The patient underwent surgical resection, and histopathologic analysis revealed anhistic membranes, corresponding to hydatid cysts. During one year of follow-up, no evidence of local or systemic recurrence was observed. In conclusion, orbital hydatidosis should be considered in the differential diagnosis of a cystic orbital tumor in pediatric patients, especially if the patient is from an endemic area.
\end{abstract}

Key words. Orbit; Neoplasms; Orbital neoplasms; Equinococcus granulosus; Echinococcosis; Zoonoses (source: MeSH NLM).

\section{INTRODUCCIÓN}

Las lesiones quísticas de la órbita pueden clasificarse según su relación con las estructuras adyacentes, por el tiempo de aparición, la etiología, o por el tipo de células que constituyen su pared, siendo estos de difícil diagnóstico debido a su similitud en sus características clínicas y radiológicas. Según su etiología, estos pueden ser traumáticos, quirúrgicos, inflamatorios e idiopáticos ${ }^{(1)}$. Las lesiones orbitarias de tipo quísticas inflamatorias son raras en la mayor parte del mundo, aunque relativamente más frecuentes en zonas tropicales, causados, principalmente, por infecciones por Echinococcus granulosus y el cisticerco de Taenia solium ${ }^{(1,2)}$.
La hidatidosis es una zoonosis parasitaria asociada, principalmente, a la especie Echinococcus granulosus ${ }^{(2)}$. EI quiste hidatídico puede localizarse en distintos tejidos, siendo los más frecuentes el tejido hepático $(70 \%)$ y pulmonar $(20 \%)^{(3)}$. La presentación orbitaria es inusual y representa, aproximadamente, 1 a $2 \%$ de los quistes hidatídicos, con más frecuencia en pacientes pediátricos ${ }^{(4,5)}$. En Sudamérica, el Perú es considerado como zona endémica, principalmente las regiones de la sierra central y sur del país ${ }^{(6,7)}$; sin embargo, no se han reportado publicaciones relacionadas al compromiso orbitario El objetivo de este reporte es describir el diagnóstico y tratamiento de un paciente en edad pediátrica con quiste hidatídico primario de la órbita, proveniente de una zona endémica de Perú.

\footnotetext{
Instituto Nacional de Enfermedades Neoplásicas (INEN). Lima, Perú

Sociedad Científica de Estudiantes de Medicina Villarrealinos (SOCEMVI), Universidad Nacional Federico Villarreal. Lima, Perú

Oftalmólogo, especialista en Oncología Ocular; ${ }^{\mathrm{b}}$ estudiante de Medicina; ${ }^{\mathrm{c}}$ médico especialista en Anatomía Patológica.

Recibido: 08/04/2017 Aprobado: 10/08/2017 En línea: 21/09/2017
}

Citar como: Cruzado-Sánchez D, Salas-Diaz S, Tellez WA, Maquera-Torres G, Serpa-Frías S. Tumor primario quístico de la órbita: a propósito de un caso de hidatidosis infantil. Rev Peru Med Exp Salud Publica. 2017;34(3):560-3. doi: http://dx.doi.org/10.17843/rpmesp.2017.343.2809 


\section{REPORTE DE CASO}

Paciente mujer de 10 años de edad, natural y procedente de zona rural del distrito de Nauminpuquio, provincia de Tayacaja, departamento de Huancavelica en Perú, quien mantenía contacto con animales domésticos, sin antecedentes patológicos de importancia, que refiere tiempo de enfermedad de dos meses, caracterizado por proptosis ocular, disminución de la agudeza visual (AV) y restricción de la motilidad en ojo derecho (OD). Fue transferida a un hospital oncológico de referencia nacional, por presentar imágenes tomográficas compatibles con tumor orbitario derecho, donde fue evaluada por el servicio de oftalmología oncológica.

Al examen clínico oftalmológico, en OD se encontró una agudeza visual (AV) de percepción de movimiento de manos (20/20000) y en ojo izquierdo (OI) se encontró una AV de 20/30. Ectoscópicamente, se evidenciaba proptosis axial indirecta irreductible del globo ocular derecho e inyección ciliar conjuntival (Figura 1). La fundoscopía del OD evidenció palidez de papila, vasos peripapilares hiperémicos, bordes difusos del disco óptico, y pliegues retinales en polo posterior.

En la tomografía espiral multicorte (TEM) de órbita se apreció una lesión tumoral de apariencia quística, ubicada en el compartimiento intraconal derecho, que comprimía y desplazaba el nervio óptico y los músculos extraoculares; la órbita y el globo ocular izquierdo presentaron características normales. Se planteó como diagnóstico: tumor quístico orbitario derecho, y como diagnósticos diferenciales al quiste epidermoide y a formas inflamatorias como la hidatidosis. Se solicitaron imágenes tomográficas de abdomen y tórax que fueron reportadas con características morfológicas normales, sin evidencia de lesiones quísticas en otros órganos. Además, se solicitaron pruebas de laboratorio como hemograma y eritrosedimentación (VSG) la cuales resultaron normales. Además, exámenes inmunoserológicos como ELISA y hemoaglutinación indirecta para este parásito, que resultaron negativos.

El tratamiento planteado fue la resección del tumor, se realizó orbitotomia medial con desinserción y reinserción del músculo recto medial, y se logró tener buen abordaje de la lesión; previo a la resección se aspiró el contenido con aguja de calibre $27 \mathrm{G}$, obteniéndose aproximadamente $7 \mathrm{~mL}$ de un líquido transparente cetrino como cristal de roca, por lo que se procedió a inyectar cloruro de sodio al $20 \%$. Posteriormente, se resecó en su totalidad la membrana quística de color blanquecina. Al análisis patológico, se reportó la presencia de membranas anhistas compatibles con quiste hidatídico y escólex del parásito (Figura 2). No se inició tratamiento antiparasitario prequirúrgico ni posquirúrgico.

En el posoperatorio, la paciente continuó con AV de percepción de movimiento de manos en $\mathrm{OD}$ y $\mathrm{AV}$ de $20 / 25$ en OI. Al año de seguimiento posterior a la cirugía, la función visual permaneció invariable, la fundoscopía del OD evidenció atrofia del nervio óptico, retina aplicada y vasos retinales adelgazados. Los exámenes de imágenes de control de la órbita, tórax y abdomen fueron descritos ausentes de lesiones tumorales.

\section{DISCUSIÓN}

Los tumores orbitarios quísticos son de difícil diagnóstico y requieren de un correlato clínico, epidemiológico, imagenológico y anatomopatológico prolijo. Se consideró como diagnóstico diferencial a las lesiones inflamatorias
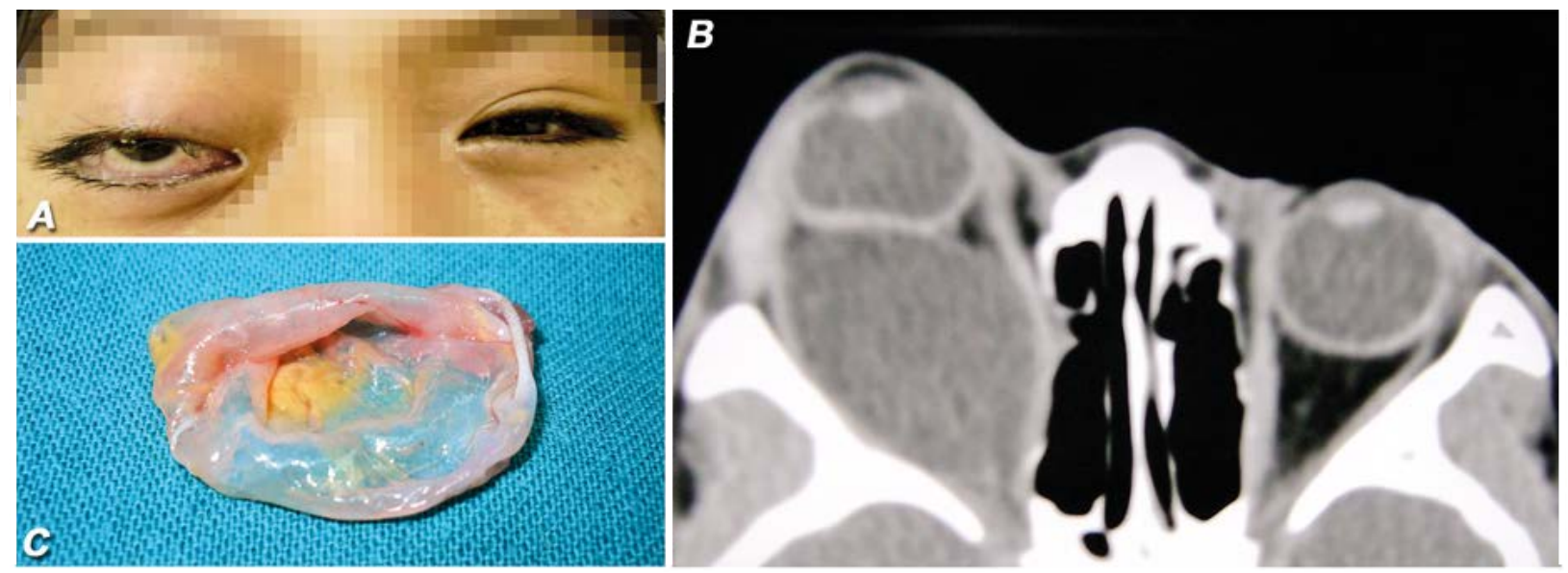

Figura 1. En (A) foto clínica de la paciente, nótese la proptosis axial del globo ocular derecho. En (B) tomografía espiral multicorte (TEM) de órbitas, se observa tumoración de apariencia quística. En (C), pieza quirúrgica de la resección tumoral orbitaria, se observa la pared del quiste hidatídico de tipo membranoso 


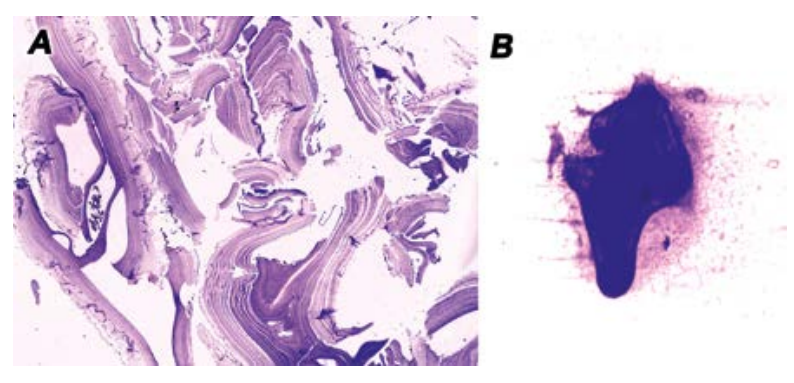

Figura 2. En (A) corte histopatológico, se muestran las láminas anhistas acelulares múltiples. En (B) imagen del escólex de Equinococcus granulosus

infecciosas como la hidatidosis, por el lugar de procedencia, la forma de presentación clínica y las características tomográficas e intraoperatorias de la lesión. El quiste dermoide también fue considerado como diagnóstico diferencial debido a su frecuencia, por ser el tumor orbitario de naturaleza epitelial más común en niños, representando aproximadamente el $89 \%$ de tumores quísticos en este grupo etario, siendo más común en la primera década de vida, en presencia de proptosis unilateral irreductible ${ }^{(1)}$, pero, a pesar de su similitud con los tumores quísticos inflamatorios, estos presentan compromiso óseo, mientras que en este caso, las estructuras óseas estaban indemnes.

En diversas publicaciones, las lesiones tumorales quísticas de la órbita en niños, de etiología inflamatoria, están asociadas a infecciones parasitarias, siendo la más frecuente la hidatidosis ${ }^{(8)}$. En nuestro medio, la infección sistémica por hidatidosis en niños es frecuente y ha sido ampliamente reportada en trabajos epidemiológicos. Cabe resaltar, que las presentaciones poco frecuentes como la órbita no se han reportado en nuestro entorno. En el Perú, la hidatidosis es un problema de salud pública, siendo las zonas endémicas, principalmente, las regiones de Puno, Junín, y Huancavelica ${ }^{(6,7)}$.

Los quistes hidatídicos orbitarios, generalmente, se encuentran en tejidos retrobulbares, ya sean externos, en los ángulos superolaterales, superomediales o dentro del cono muscular en un $60 \%{ }^{(9,10)}$. La presentación clínica consta de proptosis ocular unilateral, deterioro del movimiento ocular y de la agudeza visual en la mayoría de los casos ${ }^{(5,9)}$, lo que concuerda con el cuadro clínico que presentó nuestro paciente.

Las pruebas serológicas para lesiones únicas de la órbita por hidatidosis, no son muy específicas, por lo que resulta difícil el diagnóstico; sin embargo, se puede emplear la prueba de ELISA (Enzyme - Linked Immunosorbent Assay) y la hemaglutinación indirecta, que tienen una sensibilidad del 60 a $90 \%$, estas últimas, muchas de las veces dan resultados negativos, esto se puede deber a la localización aislada de la lesión, lo que dificulta el correcto desarrollo de anticuerpos a detectar por las pruebas mencionadas. Por lo tanto, las técnicas de diagnóstico por imágenes son muy importantes para la evaluación de estas lesiones, además de identificar posibles rupturas del quiste y la diseminación que esta conllevaría (11).

En la TEM se puede observar una masa homogénea hipodensa de bordes bien definidos e hiperdensos, en tanto que en la resonancia magnética nuclear (RMN), en la secuencia $T 1$, las lesiones se visualizan como masas hipointensas, mientras que en la secuencia T2, se observan dichas lesiones hiperintensas ${ }^{(11,12)}$; sin embargo, la RMN es una prueba de difícil acceso económicamente. En nuestro caso, se le practicó una TEM en la que se observaron las características previamente descritas, permitiendo orientar el diagnóstico y plantear el posible procedimiento quirúrgico a realizar.

El tratamiento de elección es la extirpación del quiste, donde el abordaje es variable, según distintos factores como la localización y extensión del mismo. Los tipos de abordaje que se pueden realizar son frontoorbitaria, orbitotomía lateral, medial e inferior, transcraneal, transconjuntival, rinotomía lateral, percutánea, y transmaxilar (4). En este caso se realizó una orbitotomia lateral con desinserción e inserción del músculo recto medial, esto por el tamaño tumoral y la disposición anatómica.

El diagnóstico definitivo es anatomopatológico, evidenciándose las tres capas del quiste: periquiste, ectoquiste y endoquiste ${ }^{(13)}$. El periquiste se observa bien formado por la proximidad del tejido fibroso de la órbita, el ectoquiste es acelular con apariencia de "capas de cebolla" y el endoquiste es la capa germinativa. Dentro del quiste se encuentra un líquido de tipo "cristal de roca" que puede contener al escólex, partículas de calcio e, incluso, quistes hijos ${ }^{(6)}$. Según el estudio anatomopatológico del paciente, se observaron membranas anhistas acelulares (capa cuticular externa o ectoquiste) en relación a quiste hidatídico y escólex (cabeza del parásito adulto) en un fondo de arenilla hidatídica, el líquido fue aspirado en el intraoperatorio con el fin de corroborar la lesión parasitaria, evitar una posible ruptura y potencial anafilaxia descrita en la literatura médica.

La administración de albendazol $10 \mathrm{mg} / \mathrm{kg} / \mathrm{d}$ o mebendazol $30 \mathrm{mg} / \mathrm{kg} / \mathrm{d}$ en combinación con prazicuantel durante 2 a 4 semanas antes de la cirugía, son medidas profilácticas en caso de ruptura de quiste, que se han descrito en algunos estudios ${ }^{(14)}$; en el caso presentado no se inició este tratamiento debido a los exámenes serológicos negativos. Sin embargo, cabe resaltar la importancia del tratamiento antiparasitario prequirúrgico y posquirúrgico. Aunque en nuestro caso la evolución del paciente fue favorable, siempre está presente la posibilidad de complicaciones como la ruptura del quiste, derrame del contenido y recurrencia secundaria ${ }^{(15) .}$ 
El pronóstico visual en la mayoría de estos pacientes mejora luego de haber sido tratados quirúrgicamente y con medicación antiparasitaria. Cabe mencionar que, en los casos con compromiso del nervio óptico, el pronóstico es desfavorable. En nuestro paciente, a pesar de la mejora estética y de la función parcial de la motilidad ocular, la AV se mantuvo en percepción de movimiento de manos, es decir, sin mejora visual. Esto puede ser debido al abordaje que solo fue quirúrgico, así como también, a la atrofia del nervio óptico evidenciada en los controles posquirúrgicos del paciente, el cual es un hallazgo de mal pronóstico visual. En el seguimiento posoperatorio, se debe considerar la evaluación sistémica del paciente, por la posibilidad de diseminación a otros órganos como hígado, pulmón, cerebro, entre otros ${ }^{(1)}$. Aunque en nuestro paciente, al año de seguimiento luego de la cirugía, este cursó sin evidencia de recurrencia de enfermedad infecciosa.

En conclusión, ante una tumoración orbitaria quística en pacientes pediátricos, se debe considerar como diagnóstico diferencial la hidatidosis orbitaria, sobre todo si proceden de zonas endémicas, aun tratándose de una presentación infrecuente.

Contribuciones de autoría: DCS, SDS, WAT, GMT y SSF han participado en la concepción del artículo, recolección de datos, en la redacción y aprobación de la versión final.

Fuentes de financiamiento: autofinanciado.

Conflictos de interés: Los autores declaran no tener conflictos de interés.

\section{REFERENCIAS BIBLIOGRÁFICAS}

1. Shields JA, Shields CL. Orbital cysts of childhood-classification, clinical features, and management. Surv Ophthalmol. 2004;49(3):281-99. doi: 10.1016/j. survophthal.2004.02.001

2. Rajabi MT, Bazvand F, Makateb A, Hosseini S, Tabatabaie SZ, Rajabi MB. Orbital hydatid cyst with diverse locality in the orbit and review of literatures. Arch Iran Med. 2014;17(3):207-10. doi: 0141703/AIM.0013.

3. Agudelo-Higuita NI, Brunetti E, McCloskey C. Cystic echinococcosis. J Clin Microbiol. 2016;54(3):518-23. doi: 10.1128/JCM.02420-15.

4. Somay H, Emon ST, Orakdogen M, Berkman MZ. A primary orbital hydatid cyst. J Clin Neurosci. 2012;19(6):898900. doi: 10.1016/j.jocn.2011.09.018.

5. Benazzou S, Arkha Y, Derraz S, El Ouahabi A, El Khamlichi A. Orbital hydatid cyst: review of 10 cases. J Craniomaxillofac Surg. 2010;38(4):2748. doi: 10.1016/j.jcms.2009.10.001.
6. Cucher MA, Macchiaroli N, Baldi G, Camicia F, Prada L, Maldonado L, et al. Cystic echinococcosis in South America: systematic review of species and genotypes of Echinococcus granulosus sensu lato in humans and natural domestic hosts. Trop Med Int Health. 2016;21(2):166-75. doi: $10.1111 /$ tmi.12647.

7. Chávez-Montesinos D, Salazar-Roggero Y,Piscoya A.Epidemiolgía delahidatidosis en América Latina: situación en regiones rurales endémicas. Acta Gastroenterol Latinoam. 2015;45(4):333-34.

8. Bagheri A, Fallahi MR, Yazdani S, Rezaee-Kanavi M. Two different presentations of orbital echinococcosis: a report of two cases and review of the literature. Orbit. 2010; 29(1):51-6. doi: 10.3109/01676830903278191.

9. Gómez Morales A, CroxattoJO, Crovetto L, Ebner R. Hydatidic cysts of the orbit. A review of 35 cases. Ophthalmology. 1988;95(8):1027-32.

10. Rajabi MT, Bazvand F, Makateb A, Hosseini S, Tabatabaie SZ, Rajabi MB.
Orbital hydatid cyst with diverse locality in the orbit and review of literatures. Arch Iran Med. 2014;17(3):207-10. doi: 0141703/AIM.0013.

11. Gokçek C, Gokçek A, Akif Bayar M, Tanrikulu S, Buharali Z. Orbital hydatid cyst: CT and MRI. Neuroradiology. 1997;39(7):512-5.

12. Turgut M. Hydatidosis of the Central Nervous System: Diagnosis and Treatment. Turkey: Springer; 2014.

13. Kradin RL. Diagnostic pathologic of infectious disease, 1a ed. Saunders; 2010.

14. Jimenénez-Mejías ME, Alarcon-Cruz JC, Márquez-Rivas FJ, Palomino-Nicás J, Montero JM, Pachón J. Orbital hydatid cyst: treatment and prevention of recurrences with albendazole plus praziquantel. J Infect. 2000; 41(1): 105-7.

Correspondencia: Deivy Cruzado Sánchez Dirección: Centro Oftalmológico Mácula D\&T. Carlos Ferreyros 120. Oficina 301401. San Isidro. Lima, Perú.

Teléfono: (+511) 999295482.

Correo electrónico:dcruzados@gmail.com 